\title{
El potencial de transformación y la promoción de la construcción con tierra para poblaciones vulnerables
}

\section{The transformation's potential and the earth construction's promotion in vulnerable societies}

\author{
Henrique Duarte Ferrari*, Normando Perazzo Barbosa ${ }^{* * *}$ y Carmen Ribeiro ${ }^{* * * *}$
}

Citar este artículo como: Duarte Ferrari, H., Perazzo Barbosa, N. y Ribeiro, C. (2019). El potencial de transformación y la promoción de la construcción con tierra para poblaciones vulnerables. Revista Nodo, 14(27), pp. 73-85

\section{Resumen}

A pesar de las innumerables ventajas que la construcción con tierra puede proporcionar para estos nuevos tiempos, queda latente la situación de pérdida histórica de las tradiciones populares y constructivas de las sociedades que hicieron uso de ese conocimiento, no sólo en razón de cuestiones económicas y sociales, sino también políticas y culturales. Bajo esa perspectiva, un acondicionamiento de esa práctica arquitectónica por las más diversas esferas de la realidad, la viabilidad práctica en adoptar la tierra como material constructivo en escenarios de informalidad y vulnerabilidad social sólo será posible a través de un abordaje científico/ práctico, capaz de atender esas múltiples circunstancias y motivar a los participantes para superar el estado de desinformación.

Con el objetivo de evidenciar el potencial sociopolítico que el aprendizaje de la construcción con tierra puede proporcionar, en este trabajo buscaremos demostrar, a través del análisis de casos particulares y estudios teóricos y prácticos, que el rescate de la construcción con tierra como recurso constructivo a partir de una contextualización histórica y cultural por los participantes, es capaz de contribuir a aclarar y posicionar este tema desde otra perspectiva. A partir de los análisis realizados, es posible afirmar que las relaciones conflictivas, que en otra perspectiva y dinámica pedagógica podrían limitar la real apropiación de un conocimiento que se adquiere en estos procesos colectivos, pueden volverse un potencial catalizador de transformación, con el diálogo de las diferencias y asumiendo una posición política en el proceso de aprendizaje integral y de autoafirmación de una nueva sociedad.

Palabras clave: construcción con tierra, talleres pedagógicos, tapia pisada, bahareque, emancipación social, aspecto político.

Fecha de recepción: 27 de septiembre de 2019 - Fecha de aceptación: 10 de noviembre de 2019

\footnotetext{
* Magíster en Construcción civil, Escuela de Ingeniería de la Universidad Federal de Minas Gerais -UFMG, Brasil. Correo electrónico: henrique.duarte.ff@gmail.com

** Doctor en Mecánica aplicada a las estruturas. Profesor del Centro de Tecnología de la Universidad Federal de Paraíba -UFPB, Brasil. Correo electrónico: nperazzob@yahoo.com.br

*** Doctora en Ingeniería civil y profesora de la Universidad Federal de Minas Gerais. Correo electrónico: carmencouto@ oi.com.br
} 


\section{Abstract}

However the uncountable advantages that the earth construction may provide even in current times, it is undeniable the condition of an historical lost of the traditional and popular building pieces of knowledge of the societies that made use of it, not only due to economical and social reasons, but as well as political and cultural too. Taking this in consideration, of a conditioning of those architectonic practices under the most diverse elements of reality, the practical viability of an actual adoption of the earth as a building material in informal and vulnerable contexts, will only be possible through a scientific approach that is capable of answering those multiples circumstances whilst motivating the participants in a state and perspective of self-determination and of overcoming any sort of limitations. Aiming at focusing the social and political potential that the participative and interested learning of this technology as an organized collective may promote, in this work we will have as an objective the demonstration, using of theoretical and practical studies and based in the analyses of a particular situation, that the rescue and resignification of the earth as a building medium from an historical contextualization executed by the group members is capable of contributing, evidencing and positioning this theme under a different perspective. The analyses performed made possible to assert that eventual conflictive relations that, in different perspectives and pedagogical dynamics would limit an actual appropriation of a building knowledge through a collective process, may result in itself in a potential catalyzer of transformation, from the dialogue of the differences and assuming a political position in the learning process and of self affirmation for a new society.

Keywords: earth construction, workshops, rammed-earth, cob wall, social emancipation, political aspect.

\section{Introducción}

La construcción con tierra manifiesta innumerables ventajas en los más variados aspectos económicos, sociales, políticos y culturales. Sin embargo, la tendencia de las poblaciones más vulnerables de adoptar para la construcción productos industrializados en lugar de materiales locales como la tierra, llevó a la progresiva pérdida de los conocimientos tradicionales (Dethier, 1982). Esto ocasionó la reducción del carácter cualitativo históricamente asociado a esas técnicas (Barbosa, 1999), incrementando el abandono y el actual estado de informalidad a pesar de que todavía sea adoptada por más del 30\% de la población mundial; sector en que la mayor parte no tiene condiciones para adquirir otros productos manufacturados comercialmente, según Seijo (2018). Esa situación de pérdida de las tradiciones populares y constructivas de las sociedades que históricamente hicieron uso de ese conocimiento contribuye aún más a la formación y consolidación de otros prejuicios y estigmas en relación con esa práctica de carácter milenario que, progresivamente, se van consolidando con el pasar del tiempo (Silva, 2000).

Ese cambio de perspectiva puede ser comprendido, en primera instancia, si consideramos la optimización cualitativa que los materiales industriales proporcionaron (Sharma, 2015). No obstante, este argumento solo se mantiene porque la durabilidad de las construcciones con tierra no fue tenida en cuenta, factor que puede superar los alcanzados por "la modernidad", como ya lo ha demostrado Neves (2006). De forma semejante, al argumentar que la construcción con tierra necesita de atenciones y cuidados periódicos específicos que pueden desestimular la persistencia de esta práctica en el tiempo, Sharma (2015) hace alusión a los cambios comportamentales vivenciados por la civilización en los últimos siglos. No obstante, el autor no tiene en consideración la resistencia espontánea que las personas manifiestan sobre estas técnicas en situaciones de extrema susceptibilidad, incluso con un bajo 
poder adquisitivo que les impide adquirir materiales manufacturados, como en las situaciones retratadas por Fathy (1980) y Merril (1949). Y es en ese sector de la sociedad que la renuncia de las prácticas y costumbres de la arquitectura con tierra se torna más evidente, en una negación delos conocimientos tradicionales en pro de una nueva concepción de la realidad que es impuesta sobre ellos por fuerzas político-económicas y específicamente exigencias del mercado, haciendo tabla rasa de sus conocimientos empíricos (Quintana, Grajales y Esquivel, 2013; Barbosa y Ghavami, 2014).

Bajo esa perspectiva, un condicionamiento de esa práctica arquitectónica por las diversas esferas de la realidad, la viabilidad de adoptar la tierra como material constructivo en ese escenario de informalidad y vulnerabilidad social sólo será posible, a través de un abordaje práctico y científico capaz de responder a esas múltiples circunstancias e incentivar a los participantes a superar ese estado de desinformación. Con el objetivo de evidenciar el potencial sociopolítico que el aprendizaje de ese conocimiento puede proporcionar, en este trabajo buscaremos demostrar, a través del análisis de casos particulares y estudios teóricos y prácticos, que el rescate de la construcción con tierra como recurso constructivo a partir de una contextualización histórica y cultural, es capaz de contribuir a aclarar y posicionar este tema desde otra perspectiva.

\section{Los condicionamientos socioculturales}

Las cualidades estructurales y arquitectónicas de la construcción con tierra proporcionan ventajas que no se restringen a las economías financieras obtenidas a través de la substitución de materiales industriales, si consideramos las localidades relativamente apartadas de los grandes centros urbanos y comerciales, con elevados costos logísticos. Aspectos de relevancia social y ambiental también pueden ser atendidos y exaltados tales como la armonía visual y compatibilidad del material con el ambiente y el entorno, en el que las edificaciones de tierra pueden presentar mayor integración al contexto. Otra característica relevante es el menor impacto ecológico que estas prácticas proporcionan, desde el hecho de no necesitar de combustibles fósiles hasta cualidades como el aislamiento térmico y acústico y que no demanda energía extra (Carvalho y Lopes, 2012; Pereira, Oliveira, Melo y Cavalcante, 2014). También desde el punto de vista social, la practicidad y la economía del material, así como la posibilidad de utilización de la materia prima local en la elaboración de técnicas de fácil asimilación como los ladrillos crudos con alta resistencia, se asocian a la utilización de mano de obra regional y la capacitación de un colectivo organizado. La experiencia constructiva adquirida por este segmento de la sociedad se puede transformar en actividades profesionales mediante la eficiente incorporación de la producción rural y, eventualmente, se convierte en un diferencial cualitativo en la producción artesanal local que adicionalmente puede tornarse en una posible fuente de incremento en la renta de las familias (Sertori, Folz y Ino, 2010; Folz, Sertori, Ino y Schimbo, 2009).

Todas esas características contribuyen con la reciente revalorización de este conocimiento práctico y teórico que, como nos demuestran Torgal \& Jalali (2012), se evidencia en las más diversas regiones del globo. Este retorno del interés por la construcción con tierra se debe especialmente a las cualidades funcionales extra-económicas del material que hoy retornaron y son reconsideradas en razón de las actuales preocupaciones energéticas planetarias y la desfavorable competición en costos ambientales que presenta el actual mercado de los materiales industriales, a pesar de que diferentes coyunturas vengan a demandar acercamientos y enfoques particulares. Principalmente en los llamados países "ricos" en donde la "reivindicación ecológica" puede fomentar el rescate de técnicas de construcción con tierra que se destinan principalmente a locales recreativos y ambientes que las valoricen por sus "aspectos folclóricos y arcaicos", 
mientras en los países "pobres", la utilización de la tierra como material constructivo está correlacionada con necesidades habitacionales y prioritarias (Bardou y Arzoumanian, 1981, pp. 10-32).

Esa diferencia entre estos dos contextos se evidencia en las enseñanzas teóricas y prácticas de ese conocimiento. En los sectores periféricos el aprendizaje de la construcción con tierra encuentra barreras históricamente determinadas en el sentido de que si el conocimiento no fuese debidamente comprendido y identificado, tiene la posibilidad de asumir un aspecto que va contra la naturaleza o los valores de la perspectiva de mundo de aquel que los percibe, contraste ese que provocaría según Iasi (1999) la extrañeza sobre estos nuevos saberes adquiridos. Una condición de pensamiento escéptico típica de las poblaciones marginalizadas y que, a pesar de manifestar una mirada objetiva, presenta una incoherencia al asociar morales ajenas y pasadas, con elementos de la ciencia moderna de manera "ocasional y desarticulada", como categoriza Paulo Freire (1971). De acuerdo con dicho autor, esta se constituye en una conciencia condicionada que aliena al individuo de su realidad al abstraerlo de sus componentes y precedentes históricos. Esa cuestión queda más clara cuando se considera que la transformación cultural de esas prácticas arquitectónicas es inherente a las tranformaciones socioeconómicas, culturales y comportamentales que esas sociedades vivenciaron directa $y$ indirectamente en los últimos siglos, o, como indican Bardou y Arzoumanian (1982), "las necesidades de construcción, su modo de producción, el tiempo dedicado a la construcción y las actividades de los hombres"(p. 32).

Sin embargo, lo más importante en relación con la viabilidad de apropiación del conocimiento como proceso pedagógico será el interés de los participantes en expandir sus conocimientos en el proceso de formación (Freire, 1971). O sea, en términos metodológicos, si la teorización de los cuestionamientos de la realidad va a ser abordada como una extensión de un conocimiento ajeno a ser aceptado por el grupo como "instrumento

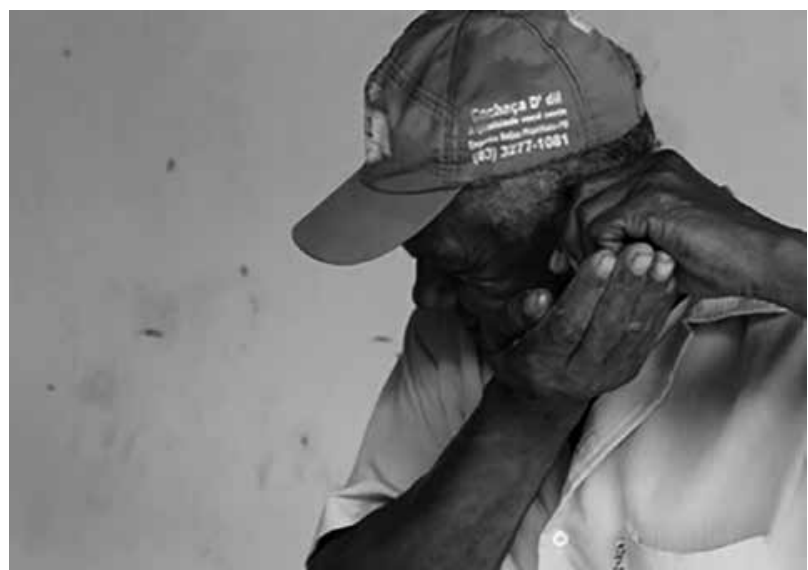

Figura 1. El señor Raimundo, poblador de Paraíba escuchando la tierra. Fuente: Lucía Esperanza Garzón.

para la adaptación del hombre" al mundo; o si esa reflexión consistirá en un cuestionamiento a ser realizado con y por el grupo, a partir del enfrentamiento con los saberes ya acumulados por él. En la Figura 1 tenemos un ejemplo de esta iniciativa, en que un participante de un taller de construcción con tierra en Brasil aprende un nuevo método empírico para comprender mejor el material con lo cual siempre trabajó.

Las iniciativas de aplicación de la arquitectura de tierra entre los sectores más bajos, invariablemente se encuentran delante del diálogo. Desde el inicio, los integrantes van a necesitar de alguna forma de gratificación asalariada por el compromiso en una dinámica con la cual no se identifican de inmediato, como relata Fathy (1980) y Merril $(1949$,$) . Ese escepticismo en relación a los nuevos$ conocimientos se da bien sea por los conceptos ya consolidados que subyugan esa metodología en relación a los productos industriales (Silva, 2000), o en razón a una desconfianza o rechazo espontáneos que se manifiestan ante los nuevos contenidos a los cuales son solamente presentados, como afirma Freire (1971).

En ese sentido, una expectativa de reacciones culturales con el aprendizaje de una nueva metodología constructiva y que propone contribuir a la restauración de esos conocimientos y saberes bajo una base científica en localidades con diná- 
micas particulares, debe tener en consideración los diversos aspectos comportamentales de esa realidad. Como en el caso de los emprendimientos autogestores de construcción con tierra que, según Dethier (1982), al rescatar procedimientos constructivos hasta entonces olvidados o desconocidos por comunidades tradicionales, posibilitan la perspectiva necesaria para un salto cualitativo en las condiciones sociales y en la capacidad de autoafirmación de esas poblaciones.

Dentro de estas experiencias de promoción organizada de la autoconstrucción, Hassan Fathy fue uno de los pioneros en promover en los últimos siglos la reinserción de la construcción con tierra abordando todas las esferas que esa filosofía engloba. Desde el aspecto económico y financiero, con la reducción del costo por la utilización de materiales locales, hasta la relación de la comunidad con el trabajo activo y asociativo de producción de su propio espacio de residencia; evidenciando los beneficios de difundir una habilidad especializada y capacitada, incluso discutiendo el carácter artístico de la experiencia del taller, donde hubo espacio para la expresión con representaciones teatrales del emprendimiento por los propios moradores (Fathy, 1980). Este último aspecto también se configura como una etapa del proceso de formación pedagógica, como categoriza Freire (1971), en la que a través de la aprehensión estética de los hechos cotidianos, es posible el desarrollo del conocer crítico de cada uno mediante el reconocimiento mutuo de los temas planteados, afirmándose como sujetos conscientes de los potenciales y de las limitaciones de sus actos como un colectivo.

Otro ejemplo de un abordaje histórico cultural de la metodología constructiva con fines sociales puede ser observado en el experimento realizado por Montaner, Cabana y Pascal (2016). Como en el caso anterior, los participantes se mostraban interesados en solucionar y satisfacer algunas de sus necesidades inmediatas (viviendas), así como en otras esferas de las relaciones allí desarrolladas, de orden político, cultural y familiar. La formación

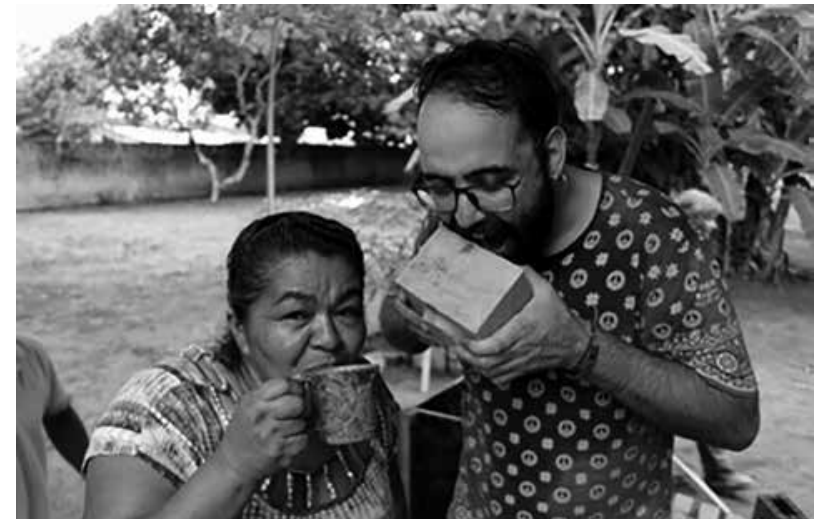

Figura 2. "Comer y beber de la tierra". Otra comunicación de la academia con las comunidades. Fuente: Lucía Esperanza Garzón.

de la conciencia crítica y de la capacidad profesional del colectivo no era concebida como una simple transferencia de contenidos acabados y de tipo abstracto. Se constituye como una actividad humanizadora que, a partir de la propia experiencia y del conocimiento de los participantes, hace posible que estos repensasen bajo una nueva perspectiva las relaciones presentes en el contexto en el cual estaban incluidos, como en la situación del taller brasileño de la Figura 2. O sea, va más allá de una cuestión de la capacidad de reivindicación de los seres humanos asociados y de la identificación de las contradicciones objetivas; y, como afirma Iasi (1999) al respecto de la formación de la autoconciencia, ese proceso de racionalización de las condiciones objetivas se torna una problemática sobre la posibilidad de acción y del interés de los individuos en la participación en la transformación de la realidad. Según este autor, esa emancipación culmina, eventualmente, en la superación de la mera opinión y de la pura constatación de la presencia aislada de los eventos por parte de los individuos, relacionándolos entonces a otros factores concretos y culturales a que sus acciones, técnicas y comportamientos están condicionados.

En este sentido, y en relación con las reivindicaciones por mejorar las condiciones habitacionales o de infraestructura, las luchas de carácter ecológico o de cuestionamiento social cuando se considera el potencial transformador de la asimi- 
lación de la construcción con tierra en colectivo, se convierten en preguntas sobre una realidad en la que sus diferencias son solamente una cuestión de escala. Como ya se ha observado, el principal factor de discrepancia es el hecho de que en los territorios que se clasifican como periferia del centro económico, los conflictos históricos apenas parcialmente superados potencializan las contradicciones internas de las transformaciones de orden mundial en los más diversos aspectos de la lucha de clases y la vulnerabilidad de las clases desposeídas.

Es decir, si por un lado la centralización de la importancia del proceso pedagógico contribuye a esclarecer las eventuales dificultades de aprendizaje del conocimiento de la construcción con tierra, por otro lado exalta algunos de los obstáculos de esta situación en contextos periféricos. La necesidad de politización de los movimientos sociales toma mayores proporciones en un contexto en que la segregación de los sectores de la sociedad no ocurre a través del mercado, en el acceso a los productos, sino que más bien es una exclusión del campo de derechos, como afirma de Oliveira (2018). Esa condición de exclusión se destaca en muchos países suramericanos, entre ellos Brasil en donde, si se analizan los datos del Instituto Brasileiro de Geografía Estadística (IBGE, 2013), la cantidad de inmuebles libres en condiciones de ser habitados supera en términos absolutos el déficit habitacional nacional, tanto en áreas rurales como en las zonas urbanas.

A través de esta aparente incoherencia no solo se destaca el hecho de que la desigualdad social, como especifica de Oliveira (2018, p. 156), no es solo una cuestión de mala gestión de recursos materiales y de capitales, sino que es también un problema que no puede ser resuelto individualmente (Guerra, Jové, Del Caz, Olmos y Camino, 2013). Eso refuerza la necesidad de un proceso pedagógico que aborde la inserción del aprendizaje de estas técnicas como un recurso viable en la sociedad capitalista actual, y a través de un posicionamiento político y colectivo. En otras palabras, en una dinámica en la cual los sujetos se identifiquen a través del propio objeto de estudio y el trabajo de la construcción con tierra. Es a partir de esta perspectiva que podemos comprender la observación de Neves (2002) al respecto de la eficiencia de la divulgación de técnicas constructivas con tierra en comunidades latinas por la red PROTERRA. La autora afirma que entre los elementos presentes en las metodologías implementadas en los diferentes países de la región a lo largo de los últimos años, el factor más determinante fue la búsqueda espontánea de conocimiento por parte de los participantes, lo que Paulo Freire llamó de "curiosidad" epistemológica (1996, p. 36).

De ahí la necesidad y la importancia en contribuir en la optimización tecnológica y metodológica de las formas autogestionarias de producción (Kapp, 2008), y de la capacidad de diálogo con otros movimientos de impronta social, entendiendo el valor de aclarar y de reforzar la resistencia para otros sectores progresistas de la sociedad, como afirma Altieri (2012). Un ejemplo es el caso de auto constructores asociados que decidieron construir sus propias viviendas con materiales no convencionales en la periferia urbana de Brasilia, vinculando la participación voluntaria de movimientos artísticos y obteniendo así repercusión nacional, conciliando el reconocimiento del conflicto de clases en el cual están insertados en un trabajo que incluye a los demás sectores de la sociedad civil (MTST, 2018).

A pesar de que la organización y la dinámica de los trabajos colectivos son diferentes entre áreas urbanas y rurales, son de extrema importancia para ambos pudiendo significar, en el caso de los últimos, la propia permanencia del agricultor en el campo, lo que puede ser facilitado por la asociación de los auto constructores, bajo intereses en común, como en el caso de la lucha por la tenencia de la tierra (Tavares y Ino., 2010; Prompt y Borella, 2010). Sin embargo, en asentamientos rurales es común ver los prejuicios en relación al uso de la tierra como material constructivo por haber sido adoptado al comienzo de la ocupación del terreno 
en la construcción de las viviendas provisionales (Cardoso, Jucksch, Da Silva, Hironaga y De Castro, 2009). Entretanto, algunas iniciativas en el sentido contrario y de mayor repercusión pueden ser mencionadas como el caso de la Escuela Nacional Florestan Fernandes (ENFF), construcción hecha con tierra por voluntarios e integrantes del Movimiento Sin Tierra (MST) a comienzos del siglo XXI, en el interior del Estado de São Paulo (Pizetta, 2007).

Buscando ampliar la viabilidad de adopción de la tierra en asociaciones organizadas en ambientes campesinos analizaremos a continuación las principales conquistas adquiridas en un reciente proceso pedagógico con grupos de orígenes diferentes. También serán propuestas aplicaciones de la tierra como material constructivo con fines diversos a los de requisitos habitacionales, como posible incremento de la productividad y como potencial medio de capacitación y especialización profesional. Nuestro fin es posicionar ese conocimiento constructivo como un posible recurso para ser utilizado por sectores de la sociedad, tanto en la construcción de sus residencias como en la formación de su autoconsciencia y en el reconocimiento de su capacidad para controlar su propia realidad.

\section{Caso Taller de construcción con tierra-Santa Rita, João Pessoa / Paraíba/ Brasil}

Teniendo en cuenta los diversos condicionamientos históricos que pueden influenciar la inserción de la arquitectura de tierra en la desigual sociedad contemporánea, una reciente experiencia en Brasil muestra otras formas de diálogo y de relaciones dentro de otro concepto de "transferencia tecnológica". En el mes de abril de 2019, la Casa de los Sueños promovió un nuevo taller de construcción con tierra coordinado por el ingeniero Normando Perazzo Barbosa y la arquitecta Lucía Esperanza Garzón de Colombia, y que fue realizado en la ciudad de Santa Rita, municipio marginal de la región metropolitana de Paraíba. En este espacio pedagógico, la comunicación entre los educandos y los educadores se dio a través de un abordaje diferente y con aspectos inusuales de la construcción con tierra. Allí se interactuó desde la motivación de los participantes y, al explorar su curiosidad, se llegó a la práctica científica para construir un mejor acercamiento al conocimiento.

Esta dinámica se retroalimentó por el carácter de diversidad que el grupo manifestaba en su composición con cerca de 50 participantes. Con una duración de tres días intensivos este taller envolvió variados sectores de la sociedad, desde profesionales autónomos y estudiantes universitarios de sectores relacionados, hasta moradores de regiones próximas y movimientos populares organizados en luchas sociales y políticas, como los integrantes del MST (Movimiento de los Sin Tierra), que participaron activamente como los demás educandos. La metodología participativa originó un desarrollo constructivo innovador para lograr una real comprensión y valorización de las tres técnicas constructivas con tierra que fueron estudiadas: el tapial ("pisé”) (Figura 3), el bahareque ("pau-a-pique"), y los Bloques de Tierra Comprimidos (BTC) producidos con la prensa Mattone (Figura 4). Prensa donada a la comunidad y que tiene otras características en su molde que ofrece un bloque tipo "lego", ladrillo crudo que facilita

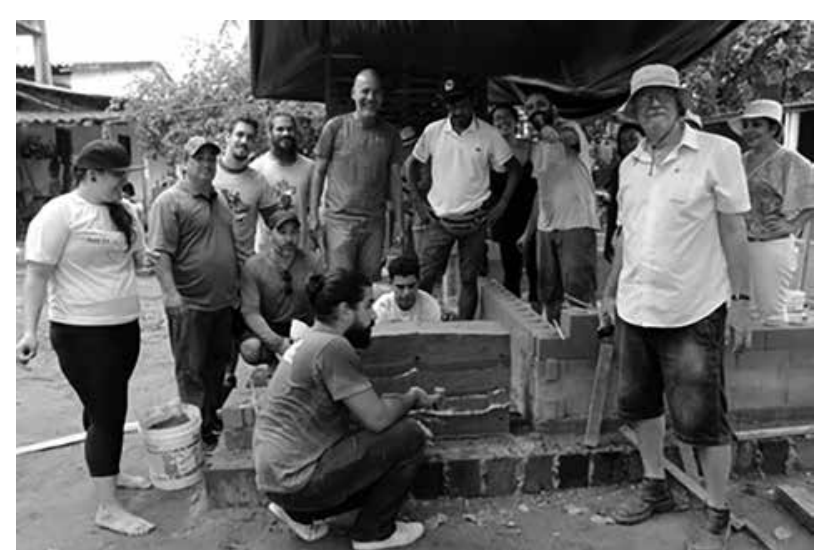

Figura 3. "Descubriendo lo evidente" con la técnica de Tapia pisada y BTC. Fuente: Lucía Esperanza Garzón. 


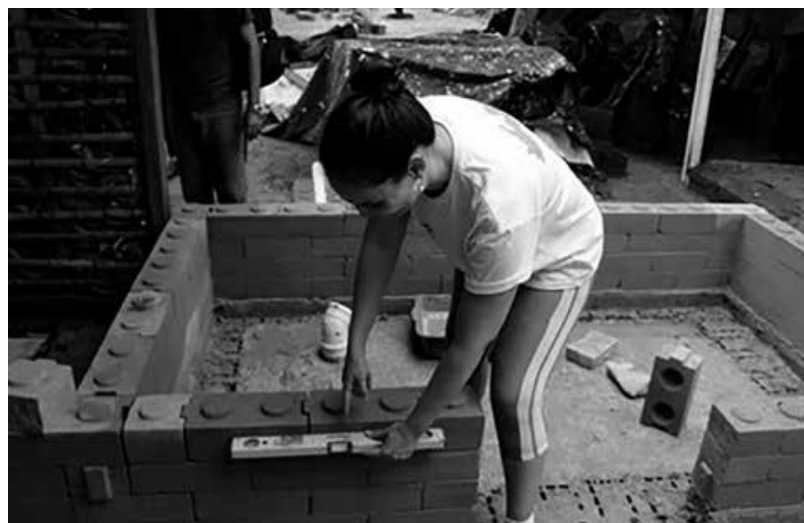

Figura 4. Mujeres universitarias aprendiendo la técnica de BTC (bloques de tierra comprimidos). Fuente: Lucía Esperanza Garzón.

el trabajo de albañilería, dejando ya terminada la pared, solo con limpiar la pared recién construida.

Una de las formas de interacción que se desplegó fue a través de la identificación entre los participantes con nuevas concepciones, donde el foco del encuentro estuvo en la valorización de las experiencias humanas y los saberes previos y a partir de este auto-reconocimiento surgieron otras características de la arquitectura con tierra y elementos culturales con los cuales podrían dialogar. La técnica constructiva con bloques de tierra comprimidos de la Figura 5, de antemano manifiesta semejanzas objetivas con la albañilería convencional, tanto en términos técnicos prácticos como de lógica y elementos teóricos, invitando a los participantes a verificarlo empíricamente.

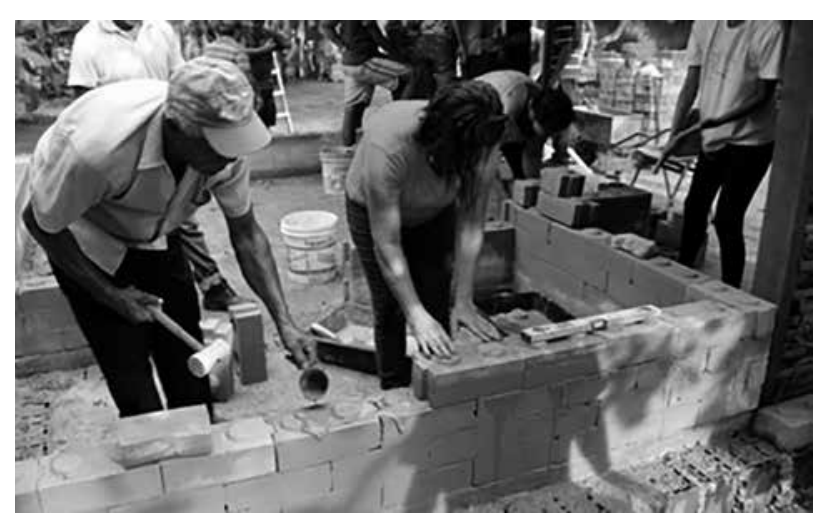

Figura 5. Aprendizaje en grupo del proceso constructivo con BTC. Fuente: Lucía Esperanza Garzón.
La construcción con el bahareque es una técnica de conocimiento cercano a la tradición y en general está presente incluso en el mismo barrio donde se realizó el taller, donde algunas viviendas han sido hechas aplicando esta técnica y fueron realizadas por algunos de los participantes del taller, lo que permitió que los asistentes tuviesen la oportunidad de aclarar dudas con el objetivo de perfeccionar sus propias construcciones. Pero, además de contribuir en aspectos de la calidad y de características higiénicos de estas edificaciones (hecho importante al considerar que la falta de revestimientos, por ejemplo, puede ocasionar la proliferación de enfermedades $y$, como consecuencia el rechazo de la tierra como material arquitectónico), la resignificación de las diferentes técnicas, así como también el aprendizaje del cada prototipo técnico, fortalecieron relaciones de confianza mutua y la disposición a la formación de un espíritu de colectividad entre los participantes.

La exigencia de una relativa sincronía entre las actividades de los miembros, de la cooperación en tareas, así como en la aplicación de los golpes (caso del tapial), o en la colocación de la arcilla para el mortero del bahareque, promovió un espacio para la autorregulación, presencia e identidad de grupo que se descubre como equipo por medio de la vivencia con las técnicas de construcción. También en razón de esta reciprocidad identitaria esa experiencia colectiva se constituyó como una confluencia de diferentes participaciones individuales que, a través del proceso educativo del trabajo, provocó la optimización de la eficiencia productiva para la obra dentro del ambiente de la alegría que produce el conocimiento entre los individuos.

Más allá, los acabados estéticos que las tres prácticas constructivas proporcionan fueron un factor que alentó y cambió paradigmas, objetivo que promovió la dinámicas del taller, generando ingeniosos diálogos que estimulan aún más el aprendizaje por parte de los participantes en relación con la arquitectura y construcción con tierra. El aspecto visual del BTC realizados con 
la máquina Mattone, que no necesita de un recubrimiento sobre la superficie exterior de los ladrillos presenta un valor agregado por su agradable aspecto y la economía en relación al trabajo del albañil y a los recursos energéticos invertidos. Las otras dos técnicas de tierra, a saber, la tapia y el bahareque, posibilitan una mayor interacción creativa y subjetiva del constructor con la apariencia de la construcción: se da de acuerdo con la selección de los tipos de materiales y sus órdenes, en el primero; y con la selección de la tierra que es versátil y permite diferentes superficies de terminación con la cal, en el segundo.

En relación con el trabajo en grupo, ese carácter artístico también estimula la sensibilidad para lograr una mejor comprensión y racionalización de las experiencias con vivencias en las cuales participaron todos los presentes. Las acciones de reconexión con ejercicios de trabajo espiritual y colectivo y las manifestaciones culturales por parte del MST transmitieron otras perspectivas de la realidad, tanto de los organizadores del taller como de los integrantes del movimiento social. En el primer caso la interacción y preparación espiritual busca evidenciar la esfera cualitativa que al momento de aprendizaje podría originar, enfatizando la necesidad de unión y empatía al prójimo, mientras las acciones místicas realizadas por los integrantes del Movimiento Sin Tierra eran capaces de compartir y dialogar su lucha social con el resto de los participantes (Figura 6).

En otras palabras, el mejoramiento y la expansión del saber constructivo con tierra que los participantes ya poseían anteriormente al taller, junto con las manifestaciones culturales y espirituales desde diferentes formas y perspectivas dibujaron una realidad y pusieron en evidencia el potencial emancipatorio de esta metodología y la pedagogía arquitectónica. Las experiencias teóricas y prácticas realizadas a través de la arquitectura de tierra integró sectores diversos de la sociedad que sumaron conocimientos complementarios a los que inicialmente se habían propuesto y que terminaron por visualizar otras maneras de rentabilidad social: por un lado, el posicionamiento

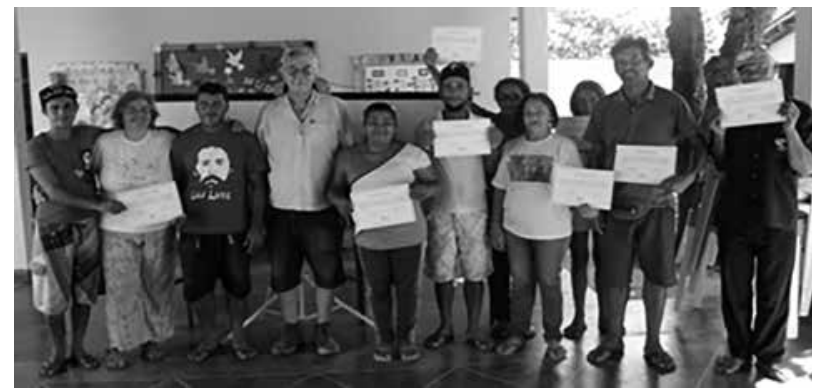

Figura 6. Entrega de los certificados a los miembros del MST. Fuente: Lucía Esperanza Garzón.

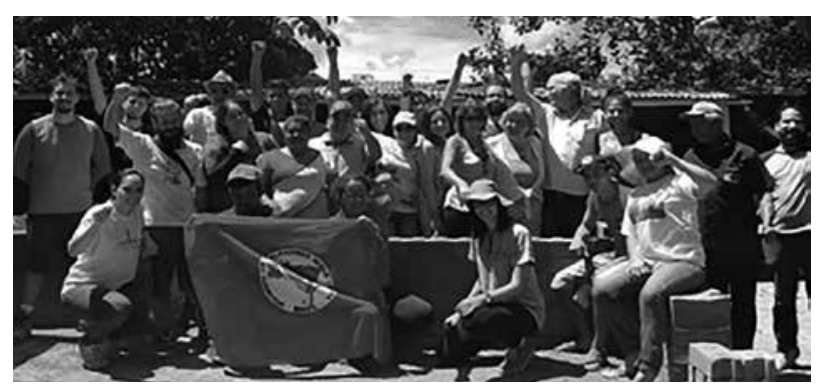

Figura 7. Cierre de las actividades del taller con todos los participantes. Fuente: Lucía Esperanza Garzón.

centralizado en aspectos políticos y culturales de las actividades realizadas ampliaron los vectores hasta el aprendizaje (Figura 6), que también son catalizadores; y por otro lado, hace posible el desarrollo y el fortalecimiento del auto-reconocimiento de las necesidades, causas y esfuerzos para un crecimiento social en los diferentes grupos con un interés universal (Figura 7).

\section{Perspectivas de aplicación y técnicas}

\section{Cúpulas y otras cubiertas abovedadas}

El hecho de que las cubiertas para viviendas de interés social pueden representar hasta un tercio del presupuesto total de la construcción (Jové, Ramón y Hernando, 2016), exige explorar métodos alternativos para cubiertas, como afirma Barbosa (1999). En la arquitectura popular de tierra en las áreas rurales, se han producido históricamente soluciones con elementos de belleza y funciona- 
lidad para las necesidades campesinas, incluso a través de la transmisión oral y fuera de los medios académicos, como en el caso de las cúpulas de adobe (Revuelta y Merino, 2014), técnica que se ha realizado por siglos en diferentes continentes y culturas.

De acuerdo con Jové et al. (2016), las bóvedas características de la región de Nubia en el actual Sudán, y, más recientemente, las cúpulas de ladrillo recargado sin formaleta que han evolucionado y traspasado continentes, también ahora denominadas Mexicanas (Aguirre, 2011), son totalmente hechas a partir de adobes, sin necesidad de madera como formaleta durante la fase de elaboración. Esta técnica consiste en una "tradición de siglos" (Fathy, 1980) que actualmente viene siendo empleada en proyectos de interés social (Jové et al., 2016). En la región de Tierra de Campos en la Península Ibérica, las construcciones de bóvedas hechas con barro fueron dominantes a lo largo de la historia humana, perdiendo su funcionalidad para los trabajos en el campo por el cambio de las necesidades y la necesidad de amplios galpones comerciales para el bodegaje de máquinas que llegaron también con el desarrollo industrial (Revuelta y Merino, 2013).

Pero el potencial de aplicación en estos contextos es relevante. El propio formato de las cúpulas ofrece un óptimo confort térmico a los usuarios debido a la presión que el aire caliente ejerce sobre las capas inferiores, pudiendo presentar una reducción de hasta $40 \%$ en la transmisión de calor externo, comparado con los techos planos. Más allá de este aspecto bioclimático el carácter estético y la practicidad de la técnica crea una concepción como estilo arquitectónico diferenciado, lo que posibilita la profesionalización rentable de aquellos que adquieren su conocimiento constructivo (Revuelta y Merino, 2014)

Con relación a las especificaciones técnicas, varios autores afirman que las cúpulas necesitan de al menos una capa de protección en la cobertura externa, y según cada lugar un refuerzo estructural para conducir mejor las aguas pluviométricas, lo que evita la acumulación en el interior de la estructura. También es posible adicionar una capa extra de tierra estabilizada que debe ser recolocada periódicamente, actividad que puede ser realizada a través de una mezcla de cal con mucílago de cactus (Aguirre, 2011).

\section{Hornos a leña}

Muchas veces las peculiaridades de la necesidad del horno a leña en el medio rural no es considerada en proyectos habitacionales que se inspiran en planos preestablecidos para el ambiente urbano (Cardoso et al., 2009). La construcción correcta de los hornos domésticos puede contribuir al mejoramiento de la higiene de los usuarios, con una cubierta de estufa y un cañón de escape por ejemplo, puede evitar el desarrollo de enfermedades características de la intoxicación por humo (Wild, 2018). Solo en Nicaragua se estima que cerca de 4 millones de personas utilizan un horno abierto en las actividades cotidianas. La construcción de un ducto de humo así como de una cámara de combustión larga para facilitar la colocación de la leña, pueden contribuir con la salud pública de esa población (Carrillo y De la Rica, 2013). Este elemento adicional de la arquitectura que hace parte del equipamiento de las cocinas es un lugar muy importante para una familia campesina $y$ sigue persistiendo como elemento constructivo en la arquitectura contemporánea que conserva la memoria y las raíces culturales de la gastronomía.

\section{Conclusiones}

Al priorizar el diálogo y el intercambio de información en el proceso de formación de los participantes, la interacción didáctica que mejora el desarrollo pedagógico y de aprendizaje de las tecnología constructivas con tierra, proporciona una comprensión de otras lógicas y realidades que transforman las condiciones objetivas de los individuos con una postura participativa, superando cualitativamente eventuales limitaciones y condicionamientos históricos. Partiendo del análisis de 
la interacción entre el conocimiento de la construcción con tierra y las necesidades de las poblaciones en una coyuntura vulnerable, es posible, a través de una profundización teórica de las técnicas y metodologías del acercamiento pedagógico, retornar a conocer las situaciones particulares de modo que contribuyan a crear un camino para su propio desarrollo, expandiendo más allá de la técnica otras formas de diálogo y identificación de esas tecnologías y conocimientos dentro de una integración a la sociedad actual; también se evidencia que los cambios de perspectiva por parte de los participantes es capaz de aclarar cuestiones determinantes para el proceso de formación en la ecología humana. O sea, la presencia interesada de los integrantes en el proceso constructivo es de fundamental importancia para la consolidación de un espíritu asociativo entre los auto constructores, incluso como proceso fundamental durante el período de ejecución de una obra y para modelar una convivencia armoniosa de los habitantes en su territorio y en la post ocupación.

Más allá de toda la experiencia profesional adquirida, incluyendo profesionales y académicos, hasta para los individuos que nunca habían ejercido cualquier tipo de función en la construcción civil, existen también aportes que quedan en la memoria incluso física y corporal de los integrantes que participan en estos movimientos que llevarán en su bagaje por la vida. La reflexión colectiva de esta posibilidad de transformación de su entorno es viable para que entre las personas asociadas se posibilite un salto cualitativo tanto en la manera como los participantes vivían y en sus conocimientos y percepciones de la realidad, así como en sus praxis constructivas. Lejos de caracterizar las familias participantes como "beneficiarias" de una política pública que pretende ser asistencialista, la construcción colectiva y autogestora con la tierra permite que los ciudadanos involucrados en el proyecto adquieran mayor responsabilidad y autonomía de su futuro y en todos los procesos que impliquen "toma de decisiones" en relación a los medios materiales para la transformación de su proprio entorno. Las relaciones conflictivas, que en otra perspectiva y dinámica pedagógica podrían limitar la real apropiación de un conocimiento que se adquiere en estos procesos colectivos, pueden volverse un potencial catalizador de transformación con el diálogo de las diferencias y asumiendo una posición política en el proceso de aprendizaje integral y de autoafirmación de una nueva sociedad.

\section{Referencias bibliográficas}

Altieri, M. (2012). Agroecologia: bases cientificas para uma agricultura sustentável. São Paulo, Brasil: Editora Expressão Popular.

Aguirre, R. (2011). Bóvedas de tierra. En: técnicas de construcción con tierra. Neves, C. \& Faria, B. (Orgs.), Bauru: FEB-UNESP, PROTERRA, pp. 26-34,.

Barbosa, N. P. (1999). Transferência e aperfeiçoamento da tecnologia construtiva com tijolos prensados de terra em comunidades carentes. En: Workshop finep de avaliação, subárea inovação tecnológica, pp. 1-11.

Barbosa, N. y Ghavami, K. (2014). Construção em terra e sustentabilidade. En: Congresso luso-brasileiro de materiais de construção sustentáveis 2014. Univ. do Minho, Guimarães, Portugal.

Bardou, P. y Arzoumanian, V. (1981). Arquitecturas de adobe. Barcelona, España: Editorial Gustavo Gili S.A.

Carrillo, E. y De La Rica, J. (2013). Fogones mejorados de adobe. Comunidad de la prusia, granada, nicaragua. En: Construcción con tierra. Patrimonio y vivienda. X Ciatti. Congreso de arquitectura en tierra en Cuenca y Villagarcía De Campos. [Online]. Valladolid: Cátedra Juan De Villanueva. Universidad de Valladolid, pp. 397-408.

De Carvalho, T. M. P. y Lopes, W. G. R. (2012). A arquitetura de terra e o desenvolvimento sustentável na construção civil. VII connepi symposium. Palmas, Tocantins, Brasil.

Cardoso, F., Jucksch, I., Da Silva, M. S., Hironaga, l. S. y De Castro, A. D. (2009). Projeto arquitetônico 
e participação popular: as dimensões formais, simbólicas e políticas da moradia e da habitação em assentamentos de reforma agrária. Campinas [SP], Brasil, Labor E engenho, 3(1), pp. 70-81.

Dethier, J. (1982). Arquitetura de terra: ou o futuro de uma tradição milenar. Lisboa: Fundação Culouste Gulbenkian

Fathy, H. (1980). Construindo com o povo (arquitetura para os pobres). São Paulo, Brasil: Editora da Universidade de São Paulo.

Folz, R., Sertori, R., Ino, A. y Shimbo, I. (2009). Construção de habitação em assentamentos rurais com geração de oportunidades de trabalho e renda. Caso: assentamento rural Pirituba II (Itapeva - SP). En: V encontro nacional e III encontro latinoamericano sobre edificações e comunidades sustentáveis, Recife, Brasil.

Freire, P. (1971). Extensão ou comunicação? Rio de janeiro, brasil: editora paz e terra, $93 \mathrm{p}$.

Freire, P. (1996). Pedagogia da autonomia: saberes necessários à prática educativa. São paulo, brasil: editora paz e terra, (coleção leitura).

Guerra, J. L., Jové, F., Del Caz, R., Olmos, P. y Camino, M. (2013). Cómo puede ayudar el conocimiento y la construcción sostenible en el desarrollo de las ciudades. El ejemplo de manta. Proyecto de cooperación internacional de la Universidad de Valladolid, España y la Universidad Laica Eloy Alfaro de Manabí, Ecuador. X Ciatti. Congreso de arquitectura en tierra en cuenca y Villagarcía de Campos 2013. [Online]. Valladolid: Cátedra Juan de Villanueva. Universidad de valladolid, pp. 353-66.

Iasi, M. L. (1999). Processo de consciência. São Paulo, Brasil: Editorial CPV.

IBGE (2013). Censo demográfico 2010. Características gerais da população. Resultados da amostra. Recuperado de: [http://censo2010. Ibge.Gov.Br/resultados.Html].

Jové, F., Ramón, L. y Hernando, D. (2016). Bóveda nubia aplicada a la construcción de un proto- tipo de vivienda de crecimiento progresivo. En: Arquitectura en tierra. Historia y renovación. XIII Ciatti. Congreso de arquitectura en tierra en cuenca y Villagarcía de Campos 2016. [Online]. Valladolid: Cátedra Juan de Villanueva. Universidad de Valladolid, pp. 87-96.

Kapp, S. (2008). A outra produção arquitetônica. En: estéticas do deslocamento. Belo Horizonte, Brasil: Associação Brasileira de Estética.

Merril, A. F. (1949). Casas de tierra apisonada y suelo-cemento. Buenos Aires, Argentina: Editorial Windsor.

Montaner, A., Cabana, H. C. y Pascal, B. M. (2016). Construyendo en tierra con las comunidades. Base-a, senegal 2011-16. En: Arquitectura en tierra. Historia y renovación. XIII Ciatti. Congreso de arquitectura en tierra en cuenca y villagarcía de campos 2016. [Online]. Valladolid: Cátedra Juan de Villanueva. Universidad de Valladolid, pp. 207-214.

MTST (2018). Criolo, Sônia Braga, Alinne Moraes e mais artistas e personalidades colocam a mão na massa para fazer casas do MTST, 2018. Recuperado de: [http://www.Mtst.Org/mtst/ criolo-sonia-braga-alinne-moraes-e-mais-artistas-colocam-a-mao-na-massa-para-fazer-casas-do-mtst/].

Neves, C. M. M. (2002). Mecanismos para transferência de tecnologia para habitação e a experiência do projeto proterra: a experiência do ceped. III Seminario Iberoamericano de Construcción con Tierra. Mendoza, Argentina.

Neves, C. M. M. (2006). O uso do solo-cimento em edificações: a experiência do ceped. En: $V$ Seminario Iberoamericano de Construcción con Tierra y I Seminario Argentino de Arquitectura y Construcción con Yierra. Mendoza, Argentina, pp. 96-107.

De Oliveira, F. (2018). Brasil: uma biografia não autorizada. São Paulo, Brasil: Editora Boitempo.

Pizetta, A. M. J. (2014). A construção da escola nacional Florestan Fernandes: um processo 
de formação efetivo e emancipatório. Revista libertas on line: pp. 24-47.

Pereira, D., Pereira, M. S., Oliveira, R. S., Melo, A. B. y Cavalcante, A. L. (2014). Projeto de uma bio alvenaria de vedação a partir de terra crua: o caso do tijolo de adobe. Revista saúde e ciência on line, 3(3), pp. 64-75.

Prompt, C. H. y Borella, L. L. (2010). Experiência em construção com terra no segmento da agricultura familiar. En: III Congresso de Arquitetura e Construção com Terra no Brasil. Campo Grande, Brasil.

Quintana, L. M., Grajales, D. R. y Esquivel, L. F. M. (2013). Casas de adobe mexicanas. Una visión contemporánea. En: Construcción con tierra. Patrimonio y vivienda. X Ciatti. Congreso de arquitectura en tierra en Cuenca y Villagarcía de Campos 2013. [Online]. Valladolid: Cátedra Juan de Villanueva. Universidad de Valladolid, pp. 47-56.

Revuelta, O. A. y Merino, F. L. (2013). Del barro a la piedra en la arquitectura auxiliar. Chozos y casetas en tierra de campos y montes torozos. En: Construcción con tierra. Patrimonio $y$ vivienda. X Ciatti. Congreso de arquitectura en tierra en Cuenca y Villagarcía de Campos 2013. [Online]. Valladolid: Cátedra Juan de Villanueva. Universidad de Valladolid, pp. 155-166.

Revuelta, O. A. y Merino, F. L. (2014). Soluciones abovedadas en la arquitectura rural de tierra de campos: los domos de adobe en chozos y casetas. Chozos y casetas en tierra de campos y montes torozos. En: Construcción con tierra investigación y documentación. XI Ciatti 2014. Congresos de arquitectura de tierra en Cuenca de Campos 2014 [online]. Valladolid: Cátedra Juan de Villanueva. Universidad de Valladolid, pp. 45-53.

Seijo, P. (2007). Cuatro décadas de investigación: normas de adobe en Perú. Revista Eco- sur. 26. Recuperado de: [http://www.Ecosur. Org/index.Php/todos-los-articulos/59ecosur-e-magazine/edicion-26-julio-2007/ 387-cuatro-decadas-de-investigacion-normasde-adobe-en-peru].

Sertori, R., Folz, R. y Ino, A. (2010). A participação de moradores na produção da habitação social rural: análise das instalações elétricas do assentamento 115 rural Sepé Tiaraju, Serra Azul - SP. En: XIII Encontro nacional de tecnologia do ambiente construído. Canela, Brasil.

Sharma, V., Vinayak, H. K. y Marwaha, B. M. (2015). Enhancing sustainability of rural adobe houses of hills and addition of vernacular fiber reinforcement. International journal of sustainable built environment, 4(2), pp. 348-358.

Silva, C. G. (2000). Conceitos e preconceitos relativos às construções de terra (Dissertação de mestrado). Fundação Oswaldo Cruz, Escola Nacional de Saúde Pública, Ministério da Saúde.

Tavares, S. F. y Ino, A. (2010). Fonte de financiamento $x$ cronograma de obras $x$ acordos de obra: impasses na construção da habitação social no assentamento rural Sepé Tiaraju, Serra Azul SP. En: XIII Encontro nacional de tecnologia do ambiente construído. Canela, Brasil..

Torgal, F. P. \& Jalali, S. (2012). Earth construction: lessons from the past for future eco-efficient construction. Construction and building materials, 29, pp. 512-519.

Wild, C. (2018). Sitio histórico -sobrevivencia de la sociedad rural y mantenimiento de su medio ambiente, 2018. Recuperado de: [https://www.Ecosur.Org/index.Php/es/ todos-los-articulos/63-ecosur-e-magazine/ edicion-24-febrero-2007/398-sitio-historicosobrevivencia-de-la-sociedad-rural-y-mantenimiento-de-su-medio-ambiente]. 